\title{
Efeitos da cinesioterapia sobre a força de preensão palmar e a qualidade de vida de um idoso longevo com esclerose sistêmica: relato de caso
}

\section{Effects of kinesiotherapy on hand grip strength and quality of life of a long-lived elder with systemic sclerosis: case report}

\author{
Mariângela DeMarco ${ }^{1}$, Rafaela Simon Myra ${ }^{2}$, Matheus Santos Gomes Jorge ${ }^{3}$, \\ Lia Mara Wibelinger ${ }^{4}$
}

http://dx.doi.org/10.11606/issn.2238-6149.v28i1p128-134

\begin{abstract}
DeMarco M, Myra RS, Jorge MSG, Wibelinger LM. Efeitos da cinesioterapia sobre a força de preensão palmar e a qualidade de vida de um idoso longevo com esclerose sistêmica: relato de caso. Rev Ter Ocup Univ São Paulo. 2017 jan.-abr.;28(1):128-4.

RESUMO: A esclerose sistêmica (ES) é uma patologia crônica e autoimune do tecido conjuntivo, que causa perda da mobilidade, da funcionalidade, da amplitude de movimento, da força muscular e do condicionamento cardiopulmonar, e prejuízo na qualidade de vida. Este estudo objetivou verificar os efeitos de um programa de intervenção fisioterapêutica na força de preensão palmar e na qualidade de vida de um idoso longevo portador de ES. Trata-se de um estudo de caso, longitudinal e intervencionista. O paciente foi atendido no Serviço de Fisioterapia Reumatológica da Universidade de Passo Fundo e o protocolo de intervenção foi baseado em um programa de intervenção cinesioterapêutica. Para a mensuração da força de preensão palmar foi utilizado o Dinamômetro Kratos ${ }^{\circledR}$ e, para avaliação da qualidade de vida utilizou-se o SF-36. Os resultados demonstraram melhora da força de preensão palmar bilateral quando se compara os resultados das avaliações pré e pós intervenção, especialmente no membro esquerdo. No que diz respeito à qualidade de vida, observou-se melhora geral nos domínios do questionário SF-36. A cinesioterapia mostrou-se um método eficaz na melhora da força de preensão palmar e da qualidade de vida de um idoso longevo com ES.
\end{abstract}

DESCRITORES: Escleroderma sistêmico; Idoso de 80 anos ou mais; Geriatria; Força da mão; Qualidade de vida; Fisioterapia.
DeMarco M, Myra RS, Jorge MSG, Wibelinger LM. Effects of kinesiotherapy on hand grip strength and quality of life of a long-lived elder with systemic sclerosis: case report. Rev Ter Ocup Univ São Paulo. 2017 Jan.-Apr.;28(1):128-4.

\begin{abstract}
The systemic sclerosis (SS) is a chronic autoimmune disease that affects the connective tissue, and causes loss of mobility, functionality, range of motion, muscle strength decreased and cardiopulmonary decline, and quality of life loss. This study aimed to verify the effects of a program physiotherapy intervention in handgrip strength and quality of life of a long-lived elderly with SS. This is a longitudinal and interventional case study. The patient was attended at the Department of Rheumatology and Physiotherapy of the Universidade de Passo Fundo and the intervention protocol was based on kinesiotherapy. For the grip strength's evaluation was used the Kratos ${ }^{\circledR}$ dynamometer and was used the SF-36 to assess quality of life. The results showed an improvement in bilateral hand grip strength when compared with pre and post intervention results, especially in the left limb. Regarding to quality of life, an overall improvement was observed in most part of the SF-36 domains. The kinesiotherapy proved to be an effective method in the improvement of the hand grip strength and quality of life of a long-lived elderly with SS.
\end{abstract}

KEYWORDS: Scleroderma, systemic; Aged, 80 and over; Geriatrics; Hand strength; Quality of life; Physical therapy specialty.

Este trabalho foi parte de um projeto de pesquisa intitulado "Força de Preensão Palmar em Idosos", aprovado pelo Comitê de Ética e Pesquisa da Universidade de Passo Fundo com registro número 345/944.

1. Fisioterapeuta graduada pela Universidade de Passo Fundo (UPF). E-mail: mariangelademarco@gmail.com

2. Acadêmica do Curso de Fisioterapia da Universidade de Passo Fundo (UPF). E-mail: rafaelasimonmyra@gmail.com

3. Fisioterapeuta e pós-graduando do Curso de Especialização em Fisioterapia Traumato-ortopédica pela Universidade de Passo Fundo (UPF). E-mail: mathjorge5@gmail.com

4. Doutora em Geriatria e Gerontologia Biomédica pela Pontifícia Universidade Católica (PUC) do Rio Grande do Sul. Docente do Curso de Fisioterapia e do Programa de Pós-Graduação em Envelhecimento Humano da Universidade de Passo Fundo (UPF).

E-mail: liafisio@yahoo.com.br

Endereço para correspondência: Rua Rio de Janeiro, 797, bairro Ipiranga, Soledade, RS, Brasil. CEP: 99300-000. 


\section{INTRODUÇÃO}

$\mathrm{N}$ o Brasil, define-se como idoso aquele indivíduo que possui 60 anos ou mais. Entre estes, encontram-se os idosos longevos, cujos mesmos possuem 80 anos ou mais de idade. Projeções futuras indicam que nos próximos 20 anos, o número de idosos brasileiros poderá ultrapassar os 30 milhões e deverá representar quase $13 \%$ da população ao final deste período ${ }^{1,2}$.

$\mathrm{O}$ aumento da população idosa demanda mudanças nos diversos segmentos que estruturam a sociedade, incluindo a saúde. A maior expectativa de vida dos brasileiros está associada ao aumento da frequência das doenças crônicas, sendo estas as principais causas do crescimento das taxas de incapacidades entre os idosos ${ }^{3}$.

A Esclerose Sistêmica (ES) é uma patologia crônica e autoimune do tecido conjuntivo caracterizada por anormalidades no sistema vascular, sistema imunológico e matriz extracelular. Histologicamente, ocorrem alterações na síntese do colágeno gerando o aumento do seu depósito e consequentes modificações tróficas na pele e em órgãos internos, acometendo, principalmente, pulmões, trato gastrointestinal, coração e rins ${ }^{4,5}$.

A etiologia é desconhecida, no entanto, acreditase que exista um envolvimento importante do sistema imunológico. É mais prevalente em mulheres, atingindo, sobretudo, indivíduos na faixa etária entre 35 e 60 anos de idade, com incidência anual de, aproximadamente, 4 a 19 indivíduos por milhão de pessoas adultas e prognóstico de vida entre 5 e 10 anos após o diagnóstico clínico ${ }^{4,6-8}$.

As manifestações cutâneas (fibrose e calcinose), vasculares (fenômeno de Raynaud e úlceras isquêmicas) e viscerais (destacando-se hipomotilidade esofágica e intestinal, refluxo gastroesofágico, pneumopatia intersticial, hipertensão pulmonar, crise renal esclerodérmica, miocardioesclerose e arritmia cardíaca) são frequentes na $\mathrm{ES}^{9}$. Tais manifestações tendem a se potencializar com o avanço da idade dos indivíduos portadores da patologia prejudicando sua qualidade de vida (QV), especialmente em aspectos físicos ${ }^{10}$.

Indivíduos com ES apresentam envolvimento das mãos, por meio do Fenômeno de Raynaud, cujo mesmo implica em ulcerações digitais, necrose tecidual, dor, infecções e incapacidade global (esta última em até $75 \%$ dos acometidos). A inflamação articular resulta em contraturas e deformidades, produzindo deficiência das mãos, comprometimento funcional e, consequentemente, impacto sobre a força de preensão palmar (FPP) $)^{8,11}$.
Os sinais e sintomas desta patologia resultam em prejuízos na mobilidade, na funcionalidade, no condicionamento cardiopulmonar e na psicomotricidade do indivíduo ${ }^{12}$. Estudos atuais referenciam que a QV de indivíduos com ES possa ser impactada negativamente em aspectos físicos e cognitivos se comparado com indivíduos sem a patologia, inclusive nos idosos ${ }^{13-15}$.

A funcionalidade pode ser mantida através da fisioterapia, sobretudo a cinesioterapia, a qual tem papel fundamental no tratamento da ES por prevenir os agravos osteomioarticulares, como os quadros de dor, as contraturas e a diminuição da amplitude do movimento e da força muscular, além de proporcionar a melhora e a manutenção da QV destes acometidos ${ }^{6,15}$.

Visto que o acometimento das mãos é frequente nesta patologia, torna-se objetivo deste estudo, descrever os efeitos de um programa de intervenção fisioterapêutica sobre a FPP e a QV de um idoso longevo portador de ES.

\section{PROCEDIMENTOS METODOLÓGICOS}

Trata-se de um estudo de caso, longitudinal e intervencionista, que faz parte de um projeto guardachuva denominado "Força de preensão palmar em idosos", aprovado pelo Comitê de Ética e Pesquisa (CEP), da Universidade de Passo Fundo (UPF) com registro número 345/944 conforme determinada a resolução 466/2012 do Conselho Nacional de Saúde (CNS).

Participou do estudo um indivíduo do sexo masculino, com idade de 85 anos, portador de ES há cinco anos, que, após concordar e assinar o Termo de Consentimento Livre e Esclarecido, foi atendido no Serviço de Fisioterapia Reumatológica da UPF, no município de Passo Fundo/RS.

Foram realizados 15 atendimentos, duas vezes por semana, de agosto a outubro de 2014 , com duração de 50 minutos cada, sendo que a primeira e a última sessão constaram de avaliação sócio-demográfica e físico-funcional, avaliação da FPP, bem como, aplicação do questionário de QV SF-36 a fim de comparação dos resultados, totalizando 17 encontros com o participante.

Para a avaliação da FPP pré e pós intervenção fisioterapêutica, utilizou-se o dinamômetro da marca Kratos ${ }^{\circledR}$, um sistema de aferição de tensão, constituído por alças fixas que não permitem a adaptação. Seu visor de leitura fica voltado para o indivíduo testado, o que permite ao mesmo acompanhar seu desempenho no momento do teste. É um instrumento que contém um sistema hidráulico fechado que mede a quantidade de força de preensão produzida por uma contração isométrica aplicada sobre 
suas alças. Essa força de preensão da mão foi registrada em quilograma-força $(\mathrm{kgf})^{16}$.

$\mathrm{O}$ indivíduo posicionou-se sentado, em cadeira padrão, sem apoio de braços, com os membros inferiores mantidos em $90^{\circ}$ de flexão de quadril e joelho com os pés apoiados no chão e, no momento em que segurava o dinamômetro, foi orientado para que não o apoiasse sobre os membros inferiores. Em relação ao membro superior, manteve-se o ombro aduzido e neutramente rodado, cotovelo flexionado a $90^{\circ}$, antebraço em posição neutra, e o punho entre $0^{\circ}$ e $30^{\circ}$ de extensão e $0^{\circ}$ a $15^{\circ}$ de desvio ulnar. Foram testadas as duas mãos, sendo três tentativas para cada mão, com intervalo de trinta segundos entre cada uma. Após foi realizada a média aritmética destas três tentativas.

A avaliação da QV foi realizada através do questionário SF-36, que foi aplicado ao paciente mediante prévia explicação do procedimento e esclarecimento de dúvidas. O SF-36 é uma escala formada por 36 itens, reunidos nos componentes físico e mental e que já foi traduzida para o português e adequada às condições sócio-econômicas e culturais da população brasileira ${ }^{17}$. O componente físico é composto pelos domínios capacidade funcional (10 itens), aspectos físicos (4 itens), dor (2 itens) e estado geral de saúde (5 itens); já o componente mental abrange os domínios vitalidade (4 itens), aspectos sociais (2 itens), aspectos emocionais (3 itens) e saúde mental (5 itens). O escore para cada domínio pode variar de uma pontuação mínima de zero ponto (que corresponde ao pior estado geral de saúde relacionado à QV) a uma pontuação máxima de 100 pontos (que corresponde ao melhor estado geral de saúde relacionado à $\mathrm{QV})^{18}$.

As avaliações da FPP e da QV do indivíduo foram realizadas por um pesquisador autônomo, cujo mesmo não implementou a intervenção fisioterapêutica neste estudo.

O programa de intervenção fisioterapêutica baseouse na cinesioterapia, sendo composto pelos seguintes procedimentos:

- Aferição da pressão arterial inicial e final;

- Alongamento muscular de modo ativo-assistido da coluna vertebral (regiões cervical, torácica e lombar e músculos da cadeia lateral do tronco), dos membros superiores (músculos extensores e flexores de punho, bíceps e tríceps braquial), dos músculos peitorais, dos membros inferiores (músculos isquiotibiais, quadríceps femorais e piriformes) durante 15 segundos para cada grupo muscular. As posições variaram em sentado, decúbito dorsal, decúbito lateral ou pé, conforme o grupo muscular alongado;
- Mobilizações articulares dos punhos, das mãos e dos dedos das mãos com o indivíduo sentado com os membros superiores sobre uma mesa, onde o terapeuta realizou as mobilizações das articulações das mãos, passivamente, com técnicas tais como tração e deslizamento;

- Ainda sentado o indivíduo realizou o fortalecimento muscular dos punhos, das mãos e dos dedos das mãos utilizando fortalecedores de dedos e mãos com carga de 0,7 que progrediu para carga 3,0 (3 séries de 3 repetições para cada dedo), fortalecedor de mãos e dedos em formato de rede (3 séries de 10 repetições) e bolinhas de borracha de carga "suave" que progrediu para carga "moderada" (3 séries de 10 repetições);

- Fortalecimento dos membros superiores (movimentos de extensão, flexão, adução e abdução de ombros, flexão e extensão de cotovelos) inicialmente de forma isométrica que progrediu para a utilização de bastão sem carga, carga de $1 \mathrm{~kg}$ e carga de $2 \mathrm{~kg}$ (3 séries de 10 repetições que progrediram para 4 séries de 8 repetições);

- Fortalecimento de membros inferiores (movimentos de flexão, extensão, adução e abdução de quadris, flexão e extensão de joelhos) inicialmente com faixa elástica cor verde que progrediu para faixa elástica azul, faixa elástica roxa, caneleira de $1 \mathrm{~kg}$ e caneleira de $2 \mathrm{~kg}$ (3 séries de 10 repetições);

- Exercícios de equilíbrio e propriocepção no balance pad de espuma (onde realizou-se descarga de peso nos membros inferiores bilateralmente que progrediu para descarga de peso unilateralmente). Após adquirir controle sobre a descarga de peso unilateral, o indivíduo progrediu para o balance pad emborrachado com superfície proprioceptiva em formato de disco (onde realizou-se descarga de peso nos membros inferiores bilateralmente que progrediu para descarga de peso unilateralmente);

- Treino de marcha entre barras paralelas que progrediu para outras superfícies como escadas e rampas;

- Exercícios respiratórios expansivos com padrão ventilatório diafragmático, que progrediu para o padrão ventilatório fracionado em 3 tempos. Estes exercícios foram associados à elevação dos membros superiores que seguraram, inicialmente, um bastão sem carga que progrediu para carga de $1 \mathrm{~kg}$ e $2 \mathrm{~kg}$, respectivamente ( 3 séries de 10 repetições);

- E, por fim, foi realizado relaxamento nos cinco minutos finais das sessões com técnicas de massoterapia nas regiões escapulares e costas. 
O número de séries e repetições para cada exercício foi determinado conforme a capacidade do participante e obedeceu a uma progressão. Além disso, todos os exercícios foram realizados em todas as sessões e foram associados a padrões respiratórios, como respiração diafragmática e inspiração fracionada, e visaram à realização de movimentos funcionais, objetivando a facilitação das atividades de vida diária. Assim, os exercícios foram direcionados para suprir as dificuldades observadas, bem como, facilitar as atividades que o voluntário mais desejava realizar.

Para a comparação dos dados obtidos, foi utilizado o Windows Microsoft Excel 2013.

\section{RESULTADOS}

As avaliações sócio-demográfica e físico-funcional demonstraram que o indivíduo era do sexo masculino, com idade de 85 anos, viúvo, tinha cinco filhos, possuía $2^{\mathrm{a}}$ grau completo e era aposentado. Fazia uso de 10 medicamentos contínuos e tinha histórico familiar de doença reumática. Não apresentava outras doenças, tais como, hipertensão arterial sistêmica, diabetes mellitus ou cardiopatia (segundo informações coletadas). Era destro e sua queixa principal era a dificuldade para realizar as tarefas que envolviam o desempenho das mãos. Ao exame físico, apresentava deformidades, restrição da amplitude de movimento e da mobilidade das seguintes articulações: dedos das mãos, punhos, cotovelos, ombros, quadris, joelhos, tornozelos e coluna vertebral em todas as regiões. Não apresentava comprometimento da articulação do quadril. Apresentava postura antálgica decorrente da dor em todos os segmentos da coluna vertebral. As atividades de vida diária como banhar-se, vestir-se, alimentar-se ou higienizar-se e marcha eram independentes (não fazia uso de dispositivos auxiliares para se locomover), embora relatasse certa dificuldade ou desconforto ao realizá-las.

A Tabela 1 apresenta os valores das médias aritméticas obtidas nas avaliações de FPP pré e pós intervenção fisioterapêutica. Pode-se observar que houve melhora da FPP nos membros superiores direito e esquerdo em 11,1 kgf e 19,0 kgf, respectivamente, após o programa de intervenção fisioterapêutica, embora tenha ocorrido maior ganho no membro superior esquerdo.

Tabela 1 - Força de preensão palmar pré e pós intervenção fisioterapêutica mensurada em quilograma-força (kgf)

\begin{tabular}{lll}
\hline Mão & Pré intervenção & Pós intervenção \\
\hline Direita & $19,0 \mathrm{kgf}$ & $30,1 \mathrm{kgf}$ \\
\hline Esquerda & $20,0 \mathrm{kgf}$ & $39,0 \mathrm{kgf}$ \\
\hline
\end{tabular}

A Tabela 2 apresenta os resultados da avaliação da $\mathrm{QV}$ pré e pós intervenção fisioterapêutica. De uma forma geral, nota-se que houve melhora na QV em todos os domínios avaliados, sendo que o aspecto físico (50\%), a dor $(42 \%)$, o aspecto social (25\%) e o estado geral de saúde $(25 \%)$ foram os que apresentaram destaque.

Tabela 2-Qualidade de vida pré e pós intervenção fisioterapêutica do questionário SF-36 mensurada em pontos

\begin{tabular}{lcc}
\hline Domínio & Pré intervenção & Pós intervenção \\
\hline Capacidade funcional & 30 & 40 \\
Aspecto físico & 0 & 50 \\
Dor & 0 & 42 \\
Estado geral de saúde & 17 & 42 \\
Vitalidade & 60 & 65 \\
Aspectos sociais & 0 & 25 \\
Aspecto emocional & 0 & 4 \\
Saúde mental & 96 & 100 \\
\hline
\end{tabular}

\section{DISCUSSÃO}

Embora saiba-se que a QV de indivíduos com ES seja prejudicada, especialmente em idosos ${ }^{13-15}$, e que a capacidade de gerar força tende a diminuir com o avanço da idade ${ }^{19}$, até o presente momento não haviam registros sobre a correlação da FPP e QV em idosos com ES. Este estudo demonstrou que com o aumento da FPP houve também melhora dos escores dos domínios da QV em um idoso longevo com ES.

Observou-se que o indivíduo, que era longevo, apresentava diminuição da FPP e dos valores dos domínios que compõem a QV na fase pré intervenção em relação a fase pós intervenção. O que poderia ser justificado pela redução de massa muscular associada ao envelhecimento, cuja mesma parece ser a principal responsável pela redução da força e potência muscular e pela consequente perda de funcionalidade em idosos. Estima-se que o envelhecimento esteja associado com $20 \%$ a $40 \%$ da diminuição da força e potência muscular aos 70-80 anos e com reduções maiores $(50 \%)$ aos 90 anos em ambos os gêneros ${ }^{19}$.

Com base nisto, um estudo foi realizado com 112 idosos do município de Passo Fundo para avaliar a FPP e compará-la com a idade, a presença ou não de doenças associadas e o sexo dos participantes. Os resultados demonstraram que $40,2 \%$ da amostra apresentava alguma doença osteoarticular associada. Na comparação entre a dominância das mãos, os indivíduos do sexo masculino apresentaram a FPP maior na mão direita em relação à mão esquerda ${ }^{20}$. No presente estudo, apesar de ser destro e apresentar alterações osteoarticulares que possam ser 
oriundas tanto pelo processo de envelhecimento, quanto pela ES, o indivíduo apresentou FPP maior na mão esquerda em relação à mão direita, tanto na fase pré intervenção (1 kgf a mais na mão esquerda) quanto na fase pós intervenção ( 9 kgf a mais na mão esquerda).

Outro estudo objetivou avaliar a FPP, com um dinamômetro da marca Kratos ${ }^{\circledR}$, em 119 idosos que apresentavam ou não dor nos membros superiores. Verificou-se que a média de FPP para os homens com dor era de $22,2 \mathrm{~kg}$ para a mão direita e $21,6 \mathrm{~kg}$ para a mão esquerda, enquanto para os homens sem dor a média era de $25,4 \mathrm{~kg}$ para a mão direita e $23,0 \mathrm{~kg}$ para a mão esquerda, demonstrando diferença estatisticamente significativa ${ }^{21}$. Nota-se que a presença de dor crônica pode interferir na FPP, uma vez que os resultados obtidos nos indivíduos com dor são semelhantes aos valores encontrados na avaliação realizada antes das intervenções fisioterapêuticas do presente estudo. Porém, quando questionado durante a aplicação do questionário SF-36 sobre a presença e influência da dor na realização das atividades, o indivíduo relata não ser esta a sua principal queixa.

Ainda, o mesmo autor demonstrou que os idosos longevos ( 80 anos ou mais), com ou sem dor nos membros superiores apresentavam FPP de 14,9 $\mathrm{kg}$ em ambas as mãos $^{21}$. Apesar disso, o indivíduo do estudo atual, que era longevo, apresentou valores de FPP superiores aos idosos longevos do estudo, mesmo na fase pré intervenção.

O envelhecimento biológico, que naturalmente provoca redução da eficácia de um conjunto de processos fisiológicos ${ }^{22}$, associado aos sinais e sintomas presentes na ES como a perda de força muscular geral e outras alterações musculoesqueléticas ${ }^{6}$ parecem ser fatores determinantes para a diminuição da FPP, o que implicaria em alterações na capacidade de realizar tarefas diárias, como vestir-se, higienizar-se e alimentar-se. A FPP que era diminuída na fase pré intervenção fisioterapêutica do indivíduo estudado pode ter influenciado negativamente a QV do mesmo, sobretudo nos aspectos físicos.

Segundo a literatura, não há um protocolo de fisioterapia específico voltado para os portadores de ES, porém verificou-se que a fisioterapia baseada em um programa de intervenção cinesioterapêutica contribuiu de forma efetiva no tratamento desta patologia, principalmente na melhora e manutenção da força muscular e da QV, gerando uma melhor execução das atividades de vida diária? .

Alguns registros na literatura citam a prática cinesioterapêutica como uma alternativa no tratamento das mãos de indivíduos com doenças reumáticas, entre elas a $\mathrm{ES}^{23,24}$. Um destes estudos envolveu 20 indivíduos com ES, submetidos a um programa de reabilitação cinesioterapêutica domiciliar monitorado $(n=10)$ e não monitorado $(\mathrm{n}=10)$. Ambos os grupos realizaram 12 semanas de intervenção e foram avaliados quanto à função da mão por meio de questionários específicos. Ao final, demonstrou-se que os programas foram benéficos para a função das mãos, contudo, o grupo monitorado apresentou melhores escores de acordo com os questionários ${ }^{24}$. Neste estudo, o programa de cinesioterapia foi elaborado de forma a poder-se monitorar as intervenções do indivíduo permitindo-lhe o bom entendimento da dinâmica dos exercícios.

O programa de intervenção fisioterapêutico utilizado neste estudo envolveu, além de exercícios resistidos, também, exercícios respiratórios com bastões, dado o acometimento pulmonar presente em indivíduos com a patologia e observou-se melhora da FPP do indivíduo estudado em 11,1 kgf no membro superior direito e 19,0 kgf no membro superior esquerdo. O que concorda com os resultados obtidos por Pinto et al. ${ }^{25}$, que recrutou 11 indivíduos com ES que foram submetidos a uma bateria de testes com diversas finalidades, incluindo a avaliação da FPP por meio do teste de força da mão. Após 12 semanas de um programa de exercícios resistidos e aeróbicos houve aumento de $11 \%$ na força das mãos.

Os resultados encontrados neste estudo, no que diz respeito à $\mathrm{QV}$, concordam com alguns autores já referenciados ao demonstrar melhora desta variável em indivíduos com ES após intervenções fisioterapêuticas, cujas mesmas envolveram exercícios diversificados, incluindo aqueles com finalidade de melhorar a $\mathrm{FPP}^{7,12,15}$.

Observou-se que o indivíduo do estudo atual apresentou melhora da QV, sobretudo nos domínios aspecto físico, dor, estado geral de saúde e aspectos sociais, após a intervenção fisioterapêutica, que envolveu cinesioterapia global e respiratória, bem como exercícios de coordenação e de equilíbrio. Semelhante aos achados realizados no estudo desenvolvido por Pedroza et al. ${ }^{12}$ que envolveu dois indivíduos com diagnóstico de ES. Ambos foram atendidos semanalmente e o programa de intervenção foi baseado em cinesioterapia global e respiratória, exercícios de coordenação e do equilíbrio e outras técnicas fisioterapêuticas como eletroterapia, hidroterapia e drenagem linfática. Ao final das sessões, verificou-se que ambos os indivíduos apresentaram evolução favorável do quadro clínico, bem como, melhora na funcionalidade e nos domínios da QV.

Ainda, outro estudo foi realizado por Jorge et al. ${ }^{15}$ que envolveu dois idosos com ES um do sexo feminino (60 anos) e outro do sexo masculino (86 anos) para analisar a dor e a QV dos mesmos pré e pós intervenção fisioterapêutica. 
Foram realizadas 15 sessões de fisioterapia, duas vezes por semana, com duração de aproximadamente 1 hora cada, baseadas em cinesioterapia que aplicou exercícios globais, respiratórios e exercícios para as mãos dos indivíduos. Os resultados demonstraram que ambos apresentaram melhora do quadro álgico e de todos os domínios da QV. $\mathrm{O}$ indivíduo do estudo atual, que era longevo, realizou um programa de intervenção semelhante ao do autor supracitado, incluindo exercícios específicos para as mãos, e apresentou melhora da QV, juntamente com aumento da FPP, após a intervenção fisioterapêutica.

Apesar de este estudo ser original, houve limitações quanto à revisão bibliográfica, o que pode apontar uma

\section{REFERÊNCIAS}

1. World Health Organization - WHO. Active Ageing - A Police Framework. A contribution of the World Health Organization to the second United Nations World Assembly on Aging. Madrid, Spain, April; 2002.

2. Instituto Brasileiro de Geografia e Estatística - IBGE. Perfil dos idosos responsáveis pelos domicílios. Brasília: IBGE; 2010 [citado 22 set. 2014]. Disponível em: http:/www.ibge. gov.br/home/presidencia/noticias/25072002pidoso.shtm.

3. Presta SA, Vidmar MF, Batista JS, Silveira MM, Wibelinger LM. Caracterização e condições de saúde dos idosos do município de Passo Fundo, no Rio Grande do Sul. Rev Bras Cienc Saúde. 2011;9(29):31-8. doi: http://dx.doi. org/10.13037/rbcs.vol9n29.1326.

4. Orlandi AC, Cardoso FP, Santos LM, Cruz VG, Jones A, Kyser C, et al. Translation and cross-cultural adaptation of the Scleroderma Health Assessment Questionnaire to Brazilian Portuguese. São Paulo Med J. 2014;132(3):1639. doi: http://dx.doi.org/10.1590/1516-3180.2014.1323621.

5. Bouer M, Chammas MC, Messina MCL. Oliveira, IRS, Cerri GG. Correlação clínica e ultra-sonográfica na esclerodermia localizada cutânea. Radiol Bras. 2008;41(2):87-91. doi: http://dx.doi.org/10.1590/S0100-39842008000200006.

6. Myra RS, Demarco M, Miotto C, Kayser B, DalMolin V, Wibelinger LM. Esclerose sistêmica: fisiopatologia e reabilitação. Rev EFDeportes. 2014;18(190):1. Disponível em: http://www.efdeportes.com/efd190/esclerose-sistemicae-reabilitacao.htm

7. Campos L, Palma R. Esclerodermia localizada: tratamento fisioterapêutico. Rev Inst Cienc Saúde. 2008;26(3):31014. Disponível em: https://www.unip.br/comunicacao/ publicacoes/ics/edicoes/2008/03_jul_set/V26_N3_2008_ p310-314.pdf. carência científica sobre a abordagem fisioterapêutica na FPP e QV em indivíduos com ES que fossem idosos, sobretudo longevos, ou do sexo masculino. Desta forma, não houveram parâmetros para comparar os valores da FPP do indivíduo estudado com outros longevos que fossem portadores da patologia.

\section{CONCLUSÃO}

O programa cinesioterapêutico implementado gerou aumento da força de preensão palmar bilateral e da qualidade de vida de um idoso longevo portador de ES.

8. Wibelinger LM. Fisioterapia em reumatologia. 2a ed. Rio de Janeiro: Revinter; 2014.

9. Sampaio-Barros PD, Zimmermann AF, Müller CS, Borges CTL, Freire EAM, Maretti GB, et al. Recomendações sobre diagnóstico e tratamento da esclerose sistêmica. Rev Bras Reumatol. 2013;53(3):258-75. doi: http://dx.doi. org/10.1590/S0482-50042013000300004.

10. Del Rosso A, Boldrini M, D'Agostino D, Placidi GP, Scapato A, Pignone A, et al. Health-related quality of life in systemic sclerosis as measured by the Short Form 36: relationship with clinical and biologic markers. Arthritis Rheum. 2004;51(3):475-81. doi: http://dx.doi. org/10.1002/art.20389.

11. Mouthon L. Hand involvement in systemic sclerosis. Presse Med. 2013;42(12):1616-26. doi: http://dx.doi. org/10.1016/j.lpm.2013.10.003.

12. Pedroza AMA, Motta MHA, Carvalho AGC, Oliveira EA, Cardia MCG, Lucena NMG, et al. Atuação da fisioterapia em pacientes com esclerodermia sistêmica: relato de casos. Rev Bras Cienc Saude. 2012;6(2):115-24. doi: 10.4034/ RBCS.2012.16.s2.16.

13. Bretterklieber A, Painsi C, Avian A, Wutte N, Aberer E. Impaired quality of life in patients with systemic sclerosis compared to the general population and chronic dermatoses. BMC Res Notes. 2014;7:594. doi: http:// dx.doi.org/10.1186/1756-0500-7-594.

14. Mao X, Sun Q. Evaluations and analyses of quality of life in 90 patients with systemic sclerosis by health assessment questionnaire-disability index. Zhonghua Yi Xue Za Zhi. 2014;94(44):3471-4. doi: http://dx.doi.org/10,3760/ cma.j.issn.0376-2491.2014.44.005. 
DeMarco M, et al. Efeitos da cinesioterapia sobre a força de preensão. Rev Ter Ocup Univ São Paulo. 2017 jan./abr.;28(1):128-8.

15. Jorge MSG, Wibelinger LM, Knob B, Zanin C. Physiotherapeutic intervention on pain and quality of life of systemic sclerosis elderly patients. Case reports. Rev Dor. 2016;17(2):148-51. doi: http://dx.doi.org/10.5935/18060013.20160034 .

16. Myra RS, DeMarco M, Pancotte J, Rodrigues D, Secchin L, Sobral D, et al. Força de preensão palmar em um indivíduo portador de lúpus eritematoso sistêmico e artrite reumatoide: um estudo de caso. EFDeportes. 2015;20(209):1. Disponível em: http://www.efdeportes.com/efd209/forca-de-preensaopalmar-em-portador-de-lupus.htm.

17. Ciconelli RM, Ferraz MB, Santos W, Meinão I, Quaresma MR. Tradução para a língua portuguesa e validação do questionário genérico de avaliação de qualidade de vida SF-36 (Brasil SF-36). Rev Bras Reumatol. 1999;39(3):143-50. Disponível em: http://www.ufjf.br/ renato nunes/files/2014/03/Valida $\% \mathrm{C} 3 \% \mathrm{~A} 7 \% \mathrm{C} 3 \% \mathrm{~A} 3 \mathrm{o}-$ do-Question\%C3\%A1rio-de-qualidade-de-Vida-SF-36.pdf.

18. Ferreira LRF, Pestana PR, Oliveira J, Mesquita-Ferrari RA. Efeitos da reabilitação aquática na sintomatologia e qualidade de vida de portadoras de artrite reumatoide. Fisioter Pesq. 2008;15(2):136-41. doi: http://dx.doi. org/10.1590/S1809-29502008000200005.

19. Garcia PA, Dias JMD, Dias RC, Santos P, Zampa CC. Estudo da relação entre função muscular, mobilidade funcional e nível de atividade física em idosos comunitários. Rev Bras Fisioter. 2011;15(1):15-22. doi: http://dx.doi.org/10.1590/ S1413-35552011000100005.

Recebido em: 24.02.16

Aceito em: 11.03.17
20. Dresch DR, Taucher V, Wibelinger LM. Força de preensão palmar em idosos. Rev EFDeportes. 2014;19(194):1. Disponível em: http://www.scielo.br/pdf/rbfis/v16n6/ aop057_12_sci1362.pdf.

21. Wagner PR, Ascenço S, Wibelinger LM. Força de preensão palmar em idosos com dor nos membros superiores. Rev Dor. 2014;15(3):182-5. doi: http://dx.doi.org/10.5935/18060013.20140040 .

22. Azevedo LF, Costa-Pereira A, Mendonça L, Dias CC, CastroLopes JM. Epidemiology of chronic pain: a population-based nationwide study on its prevalence, characteristics and associated disability in Portugal. J Pain. 2012;13(8):773-83. doi: http://dx.doi.org/10.1016/j.jpain.2012.05.012.

23. Pani D, Barabino G, Dessì A, Tradori I, Piga M, Mathieu A, et al. A Device for Local or Remote Monitoring of Hand Rehabilitation Sessions for Rheumatic Patients. IEEE J Trans Eng Health Med. 2014;2(1):2100-111. doi: http:// dx.doi.org/10.1109/JTEHM.2014.2299274.

24. Piga M, Tradori I, Pani D, Barabino G, Dessì A, Raffo $\mathrm{L}$, et al. Telemedicine applied to kinesiotherapy for hand dysfunction in patients with systemic sclerosis and rheumatoid arthritis: recovery of movement and telemonitoring technology. J Rheumatol. 2014;41(7):132433. doi: http://dx.doi.org/10.3899/jrheum.130912.

25. Pinto AL, Oliveira NC, Gualano B, Christmann RB, Painelli VS, Artioli GG, et al. Efficacy and safety of concurrent training in systemic sclerosis. J Strength Cond Res. 2011;25(5):14238. doi: http://dx.doi.org/10.1519/JSC.0b013e3181d6858b. 